\title{
First detection of helium emissions in RR Lyrae ${ }^{\star}$
}

\author{
D. Gillet ${ }^{1}$, N. Fabas ${ }^{2,3,4}$, and A. Lèbre ${ }^{4}$ \\ ${ }^{1}$ Observatoire de Haute-Provence - CNRS/PYTHEAS/Université d'Aix-Marseille, 04870 Saint-Michel l'Observatoire, France \\ e-mail: denis.gillet@oamp.fr \\ 2 Instituto de Astrophysica de Canarias, 38205 La Laguna, Tenerife, Spain \\ e-mail:nfabas@iac.es \\ 3 Departamento de Astrofísica, Universidad de la Laguna, 38205 Tenerife, Spain \\ ${ }^{4}$ LUPM - UMR 5299 - Université Montpellier II/CNRS, 34095 Montpellier, France \\ e-mail: Agnes.Lebre@univ-montp2.fr
}

Received 3 December 2012 / Accepted 28 January 2013

\section{ABSTRACT}

\begin{abstract}
Context. During the past 100 years, helium emission lines have never been observed in RR Lyrae stars, especially in RRab-type ones where strong shock waves are nevertheless present in their atmosphere. Only recently have the observation of He I emission lines in 10 RRab stars and the detection of very weak He II emission in 3 of them been reported. All these stars are Blazhko stars, while helium emission has never been detected in non-Blazhko stars.

Aims. We investigate the physical origin of the presence (or absence) of helium emission in the spectra of RR Lyrae stars, using a recent explanation of the Blazhko effect based on the interplay between two shocks occurring during each pulsation cycle.

Methods. From high-resolution spectra collected with ESPaDOnS at CFHT, we report for the first time the observation of He I emission lines in the brightest RR Lyrae star of the sky, RR Lyr. Our observation was done just after a Blazhko maximum. We have also very likely detected He II emission.

Results. The presence of He I and He II emission lines is directly connected to the shock intensity. Helium emissions only occur during the strongest amplitude phase of Blazhko stars in which an amplification mechanism of the shock intensity arises. This kind of hypersonic shock has not yet been reported in non-Blazhko stars because in this picture the intensity of their shocks is not amplified. Conclusions. The occurrence of helium emission is a direct consequence of the Blazhko effect. The greater the Blazhko amplitude, the greater the shock intensity, and therefore emission lines of helium increase. Considering the spectroscopic features observed (or not) in the RR Lyrae stars, we can define three hydrodynamic regimes: (a) the supersonic regime in which only hydrogen emissions are visible such as in non-Blazhko stars; (b) the weak hypersonic regime where the He I emission lines are also observed; and (c) the strong hypersonic regime with the additional observation of He II emission lines. These two last regimes only occur in Blazhko stars.
\end{abstract}

Key words. shock waves - stars: variables: RR Lyrae - stars: individual: RR Lyr - stars: atmospheres

\section{Introduction}

Because it is the brightest RR Lyrae star of the sky, RR Lyr was one of the most observed RR Lyrae during the last century both in spectroscopy and photometry. Its light curve exhibits a (variable) period around 0.5667 days or, equivalently, $13.6 \mathrm{~h}$ (the pulsation period), but it also presents light modulations with a (variable) period around 39 days. These amplitude and phase modulations are well known as the Blazhko effect (Blazhko 1907). Its physical origin has remained a mystery up to the present day, but interesting solutions have been recently proposed: by Stothers (2006): changes in the structure of the outer convective zone, due to an irregular variation of the magnetic field; Buchler \& Kollath (2011): fundamental mode destabilized by a 9:2 resonant interaction with the 9th overtone and Gillet (2013): interaction of two shock waves in connection with the dynamics of atmospheric layers.

During a pulsation cycle in RR Lyrae stars, there are three apparitions of hydrogen emission (Preston 2011). The so-called "first apparition" was discovered by Struve $(1947,1948)$ and

* Based on observations obtained at the Canada-France-Hawaii Telescope (CFHT), which is operated by the National Research Council of Canada, the Institut National des Sciences de l'Univers of the Centre National de la Recherche Scientifique of France, and the University of Hawaii.
Struve \& Blaauw (1948). It occurs just before the luminosity maximum. Struve proposed that the upper part of the atmosphere falls towards the star, while the deeper layer moves outwards. The emission lines may be due to the energy liberated from the collision of these two moving parts of the atmosphere. For the first time, Abt (1959) attributed the origin of these emissions to the presence of the hot emitting layer behind an outwardmoving shock front. He proposed a simple shock model in which the wave is first subsonic at the mean depth of formation of metallic lines and then becomes supersonic higher in the atmosphere where the cores of the Balmer lines are formed. He estimated that hydrogen gas is completely ionized in the hot emitting layer. For temperatures of the hot region behind the shock of $4 \times 10^{4}-10^{5} \mathrm{~K}$, helium is expected to be completely ionized. Consequently, Abt expected to see neutral and ionized helium lines in emission, however no helium emission has been observed.

The first detailed spectroscopic study of RR Lyr was done by Preston et al. (1965) during the whole Blazhko cycle, but this investigation was limited to the phases of rising light within the 13.6 hour-long pulsation cycles. Preston confirms Struve (1947) suspicion that H-emission strength varies within the 41 days Blazhko cycle. Indeed, it is maximum at the maximum of the Blazhko amplitude. The visibility of the doubling of the $\mathrm{H}$-absorption is also roughly in phase with the 
strength of H-emission. Preston et al. (1965) have found that a shock wave occurs during the rising light. Its intensity and velocity increase as the shock propagates outward in the atmosphere. During the Blazhko cycle, the critical level of shock formation moves up and down in the atmosphere. In addition to the now well known emission occurring just before the luminosity maximum, Gillet \& Crowe (1988) discovered a small blueshifted emission component within the large $\mathrm{H} \alpha$ absorption of X Ari (a non-Blazhko RR Lyrae star) and RR Lyr. This small emission appears during the bump, i.e., before the luminosity minimum. Gillet \& Crowe (1988) attributed this "second apparition" to a secondary shock produced when outer atmospheric layers in ballistic infall overtake inner deep layers. For RR Lyr, this secondary emission was not observed at the Blazhko minimum phase $(\psi=0.50)$ but only just before the maximum $(\psi=0.97)$. This means that an amplitude threshold may exist to induce this secondary shock.

Recently, Preston (2011) have found another hydrogen emission episode during the pulsation cycle (the "third apparition"), which had never been detected before. This weak emission shoulder appears within the $\mathrm{H} \alpha$ absorption near phase 0.2 , after the maximum light. Its physical origin has not yet been explained.

Schwarzschild (1952) was the first astronomer to propose that a shock wave moving throughout the atmosphere during the rising light of each pulsation cycle can explain the presence of emission lines in the population II Cepheids. The first shock model was proposed by Whitney (1956), but the prediction of the occurrence of He I emission lines was discussed by Wallerstein (1959). Unlike Whitney, and by taking into account the energy of ionization into Rankine-Hugoniot relations, Wallerstein found that a shock front with a velocity higher than $80 \mathrm{~km} \mathrm{~s}^{-1}$, has enough energy to ionize hydrogen completely, He I, but only a small fraction of He II. Thus, the observation of helium emission lines seems quite possible. However, during the next halfcentury following Wallerstein's theoretical prediction, no one observed this emission until Preston's discovery in 2009. How can this incredible delay be accounted for?

During the course of an echelle spectroscopic survey at Las Campanas Observatory, Preston $(2009,2011)$ unexpectedly found $\mathrm{He} \mathrm{I}$ lines in emission and/or absorption during rising light $(0.8<\varphi<1.0)$ in 10 Blazhko RRab stars. The strongest line was He I $\lambda 5875.66$ (D3), which appears first as a pure emission feature unlike the Balmer lines for which the absorption is always present. In addition, Preston detected very weak He II emission at $\lambda 4685.68$ in three Blazhko stars observed during largeamplitude Blazhko cycles in which metallic line doubling also occurred. He I and He II emissions were not detected in nonBlazhko stars. Preston (2009) only speculates that helium emissions may be a manifestation of the different shocks that occur during an RR Lyrae pulsation cycle such as predicted by Fokin \& Gillet (1997).

In this paper, we present for the first time the observation of helium emission lines in RR Lyr itself, a Blazhko variable. In Sect. 2 we describe observational and data reduction processes. The detection of helium line emission is presented in Sect. 3. The physical origin of helium emissions is specified in Sect. 4, while the connection with the Blazhko effect is discussed in Sect. 5. Finally, some concluding remarks are given in Sect. 6.

\section{Observations and data analysis}

On July 4, 2011, we used the ESPaDOnS spectropolarimeter at the CFHT (Mauna Kea, Hawaii) in order to collect high-resolution spectra of RR Lyr during almost five continuous hours selected around the maximum luminosity of one of its pulsation cycles of $13.6 \mathrm{~h}$. However, our observations cover less than $50 \%$ of the period, and focus particularly on some interesting phases of shock propagations: emergence from the photosphere, propagation throughout the lowest atmospheric layers, acceleration and propagation throughout the upper layers. During these phases, the spectra of RR Lyr are known to exhibit specific signatures, such as emissions on the wings of Balmer hydrogen lines or large velocity variations and broadening of metallic lines, because the shock wave imprints ballistic motion in the lowest atmospheric layers Chadid \& Gillet (1996a,b).

Using ESPaDOnS in its spectropolarimetric mode, we obtained 54 consecutive Stokes I spectra over a large part of the visible domain (from 3800 to $10100 \AA$ ) and with a resolution power of about 65000 . With a complete spectropolarimetric sequence $(U, Q$, and $V)$ obtained within seven minutes, the temporal resolution is also excellent for a 4-m class telescope, and the signal-to-noise $(\mathrm{S} / \mathrm{N})$ is about 180 at $\mathrm{H} \beta$ and about 210 around the sodium lines (at $590 \mathrm{~nm}$ ). The data were reduced with the Upena package at the CFHT, using the Libre-ESpRIT software (Donati et al. 1997). This software performs classical operations, such as bias subtraction, flat-fielding, masking of bad pixels, wavelength calibration, and spectrum extraction, plus extraction of the polarimetric information.

The phases of our 54 spectra have been computed with an ephemeris involving the period $P=0.56686776$ days and the reference Julian date from a previous maximum light of 2455743.588, as indicated in the GEOS RR Lyr database (Le Borgne et al. 2007):

$\operatorname{HJD}(\max$. light $)=2455743.588+0.56686776 E$.

Hereafter the pulsation phase is noted by $\varphi$. Then, over a typical 13.6-h pulsation cycle, our observations cover the phases from $\varphi=0.741$ to 1.17 . So as to visualize the time variation of line profiles over this phase interval, we have developed a PYTHON tool allowing the graphic display of all our 54 spectra normalized to the continuum and centered on a specific spectral line. The velocities are presented in the rest frame of RR Lyr with a star velocity $V_{*}$ with respect to the solar system barycenter of $-73.5 \mathrm{~km} \mathrm{~s}^{-1}$ (Chadid \& Gillet 1996a).

The determination of the Blazhko phase of our observations is not easy. Indeed, because the Blazhko period varies, we must know an epoch of the maximum light amplitude close to our observations. The best available ephemeris can be estimated from the Q2 Kepler data (Kolenberg et al. 2011). Using the variation in the amplitude of the Fourier parameter $A_{1}$ and the value of the Blazhko period found by these authors, we obtain

$\mathrm{HJD}(\max$. light ampl. $)=2455040.0+39.1 E$.

Hereafter, the Blazhko phase is noted by $\psi$. The maximum light amplitude corresponds to July 28, 2009. Thus we obtain a Blazhko phase $\psi=0.04$, i.e., just after the maximum light amplitude in which the strongest shocks are expected to occur. However, there are 18 Blazhko cycles between the chosen maximum light amplitude of the Q2 Kepler observations (June 19-September 16, 2009) and ours. Moreover, using times of light maximum of RR Lyr collected by the GEOS RRLyr web database $^{1}$, we find that a Blazhko maximum light amplitude occurred approximately a week before our observations. This corresponds to $\psi=0.18$. Finally, we estimate that our observations

1 The GEOS database is freely accessible on the internet at http://rr-lyr . ast.obs-mip. fr/dbrr/. 


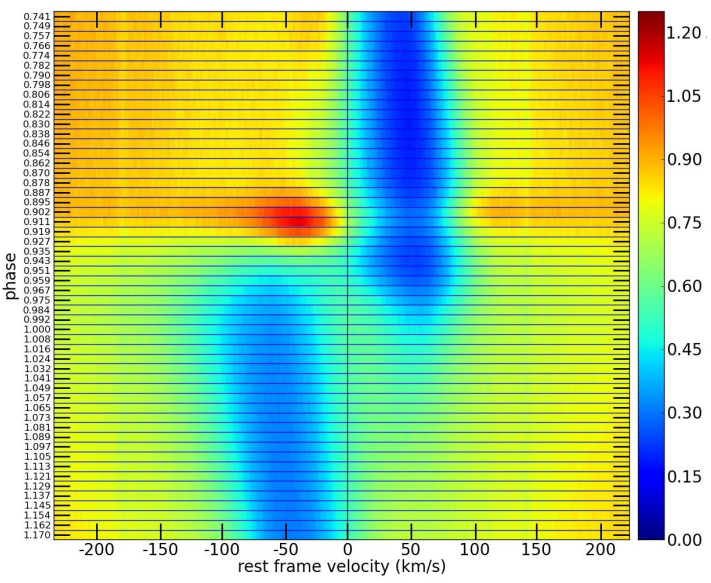

Fig. 1. Time series of $\mathrm{H} \alpha$ lines of RR Lyr, ordered by increasing phase from top to bottom. Time series of ESPaDOnS spectra are interpolated to provide a 2-dimensional map of the $\mathrm{H} \alpha$ profile in the $[-200,200] \mathrm{km} \mathrm{s}^{-1}$ velocity range. Velocities are given in the stellar rest frame with positive velocities corresponding to inward motion (toward the photosphere). The spectrum pulsation phase is indicated on the left edge. On the right side, the color bar indicating the flux is from 0.0 to 1.2 .

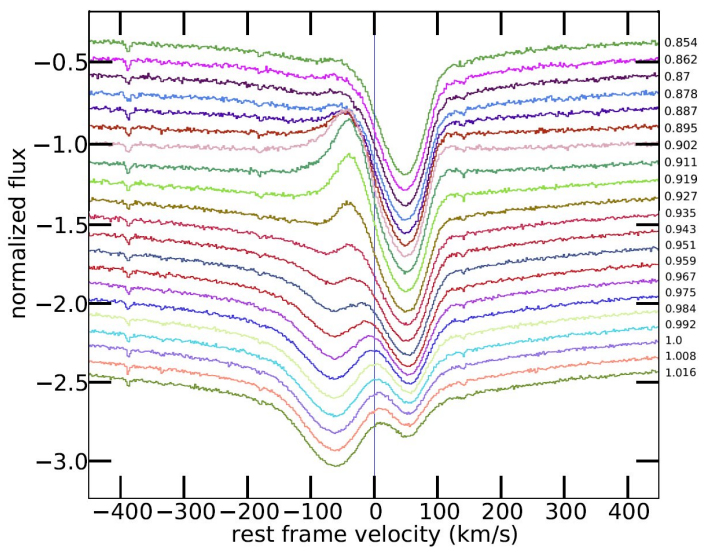

Fig. 2. Evolution of the $\mathrm{H} \alpha$ line profile of RR Lyr. The pulsation phases are indicated on the right side. The vertical line represents the zero velocity in the stellar rest frame.The profiles are arbitrarily shifted in flux.

(July 4, 2011) took place a few days after a maximum light amplitude, i.e., around $\psi \simeq 0.1$.

\section{Observation of hydrogen and helium emissions in RR Lyr}

Figures 1 and 2 show the variation in the $\mathrm{H} \alpha$ line profile from $\varphi=0.741$ to 1.170 for the $2 \mathrm{D}$ plot (Fig. 1) and from $\varphi=0.854$ and 1.016 for the 1D plot (Fig. 2). The blue emission shoulder (called the "first apparition" by Preston 2011) reaches its maximum intensity at $\varphi=0.911$. It is visible until $\varphi=0.927$ when its intensity is just below the continuum and it is followed by the subsequent double absorption lines. As discussed by previous authors (see introduction), the profile variations of the double absorption closely follow the so-called Schwarzschild's mechanism (1952). This characteristic absorption line doubling is the consequence of the propagation of a shock wave throughout the atmospheric layers. The shock is a progressive wave producing a phase delay from the bottom to the top of the reversing layer.

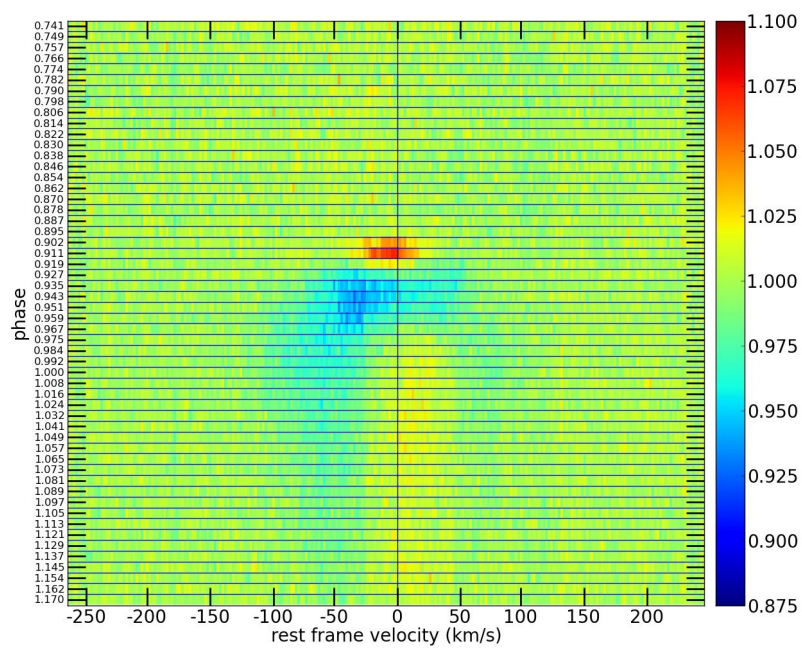

Fig. 3. Time series of the He I $\lambda 5875.66$ (D3) lines of RR Lyr, showing the lack of absorption before the sudden appearence of the emission (0.902-0.919)

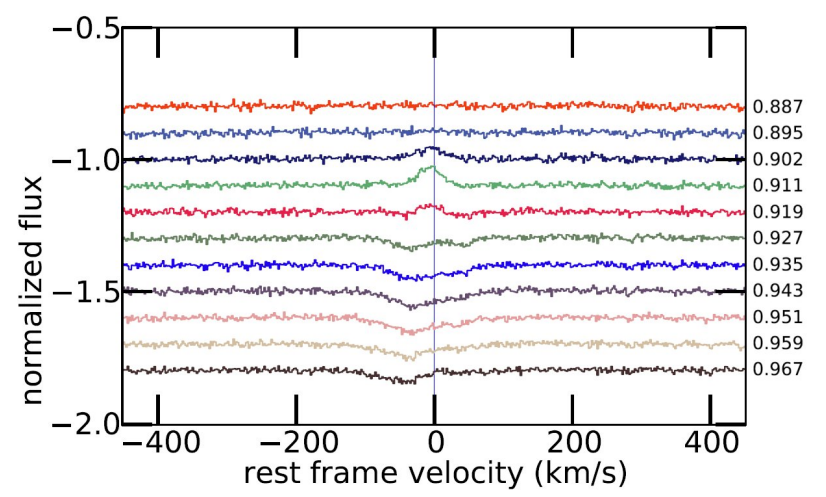

Fig. 4. Evolution of the He I $\lambda 5875.66$ (D3) line profile of RR Lyr. The emission is visible on three consecutive spectra $(0.902,0.911$, and 0.919)

Just after the first apparition of the $\mathrm{H} \alpha$ emission shoulder (at $\varphi=0.887$ ), a sudden onset of the He I $\lambda 5875.66$ (D3) emission appears at $\varphi=0.902$. It is weakly blueshifted $\left(-6 \mathrm{~km} \mathrm{~s}^{-1}\right)$, while the $\mathrm{H} \alpha$ emission component is more blueshifted $\left(-43 \mathrm{~km} \mathrm{~s}^{-1}\right)$. Figures 3 and 4 present the variation in the HeI D3 line profiles, respectively as a 2D plot (from $\varphi=0.741$ to 1.170) and from a 1D plot (from $\varphi=0.887$ to 0.967 ). From these figures, nothing was visible (no absorption) before the appearance of He I emission. The maximum He I emission is near $7 \%$ at $\varphi=0.911$ and occurs at the same time as the maximum $\mathrm{H} \alpha$ emission $(I=27 \%)$.

It seems (see Fig. 4) that a Schwarzschild's mechanism (absorption line doubling) follows the emission, but the moment in which the blueshifted absorption component is weaker than the redshifted one is not visible. This is certainly the consequence of the lack of time resolution for our data. As reported by Preston (2011), we also observe the reapparance of a very weak D3 emission after maximum light until approximately $\varphi=1.1$. For RR Lyr, the emission intensity is near $2 \%$ with respect to the continuum (see Fig. 4). Unlike our work on RR Lyr, Preston (2009) observed the complete line profile evolution of the Schwarzschild's mechanism for RV Oct (see his Fig. 1). With spectra taken at intervals between 3 and 10 min and with a similar pulsation period than RR Lyr, the time resolution used by Preston was up to twice as good as ours. 


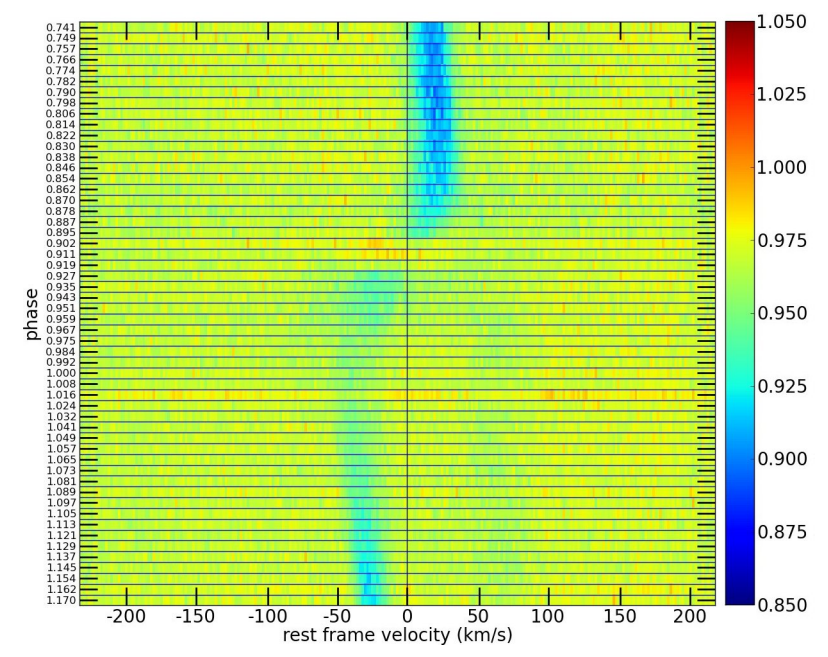

Fig. 5. Time series of the He I $\lambda 6678.16$ lines of RR Lyr. A redshifted absorption is present before the appearance of the very weak emission $(\varphi=0.911)$.

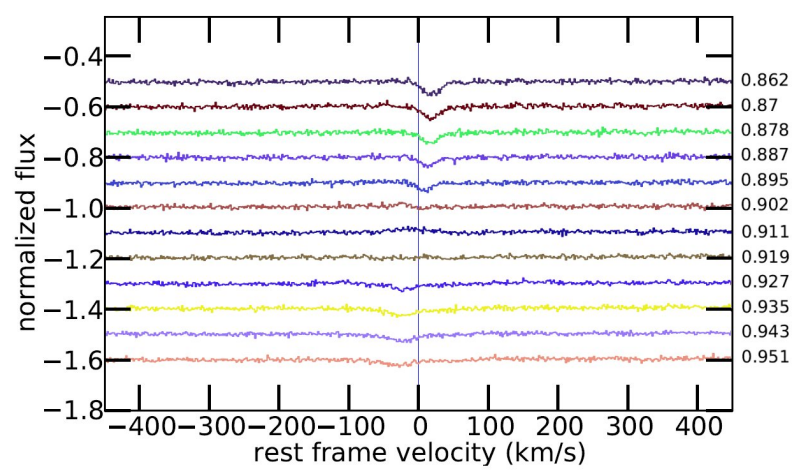

Fig. 6. Evolution of the He I $\lambda 6678.16$ line profile of RR Lyr. The weak emission is at $\varphi=0.911$.

The second helium line for which we have also detected an emission is He I 26678.16 (singlet), and Figs. 5 and 6 display its profiles variation through $2 \mathrm{D}$ and $1 \mathrm{D}$ plots, respectively. Its intensity was lower ( $2 \%$ at its maximum, i.e., at $\varphi=0.911)$ than for He I $\lambda 5875.66$ (7\%). However, unlike He I $\lambda 5875.66$ (triplet), a redshifted absorption component is visible from the beginning of our observing run (i.e., from $\varphi=0.741$ ). This is due to the partial metastability of the lower level of the triplet line. As far as W Virginis is concerned, Lèbre \& Gillet (1991) and Kovtyukh et al. (2011) observed the same behavior between these two red helium lines. The He I 76678.16 deep absorption decreases until the apparition of the emission. Then, a weak blueshifted absorption component comes into effect with an increasing intensity until our final observing phase $(\varphi=1.170)$. This absorption component returns to the zero velocity during the infall phase of the atmosphere.

If we analyze our observations to their limits, we have likely detected a very weak He II emission at $\lambda 4685.68$ at $\varphi=0.911$ and 0.919 (Figs. 7 and 8 for the 2D and 1D plots). Its intensity above the continuum is between $0.2 \%$ and $0.4 \%$ depending on the phase. The "strongest" He II emission occurs when the $\mathrm{H} \alpha$ emission is also at its maximum $(\varphi=0.911)$, i.e., when the main shock reaches its maximum intensity. The observational time scale is very short ( $15 \mathrm{~min})$. This quick phenomenon has been observed before by Preston (2011) in the spectrum of three Blazhko stars. However, remarkably, it has never been observed

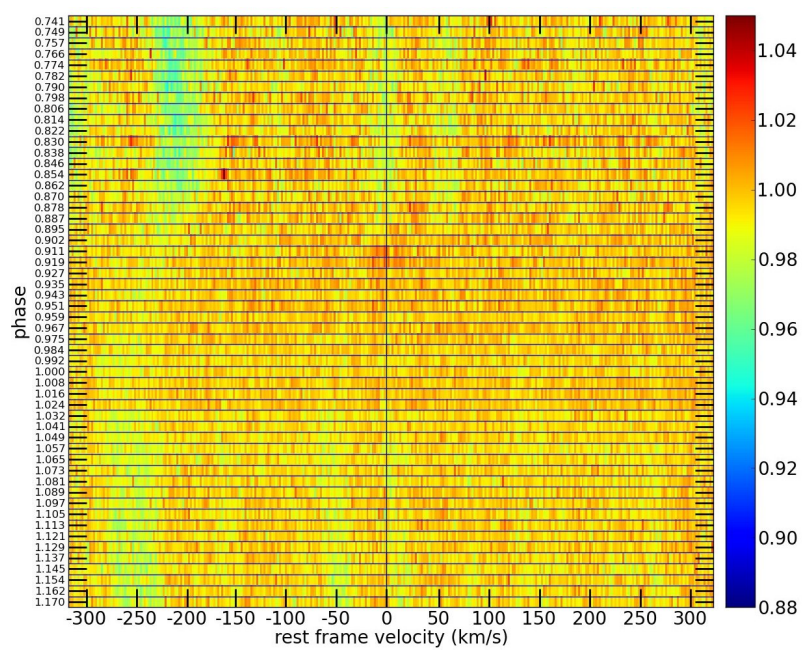

Fig. 7. Time series of the He II $\lambda 4685.68$ lines of RR Lyr. A very weak emission at $\varphi=0.911$ and 0.919 may be detected.

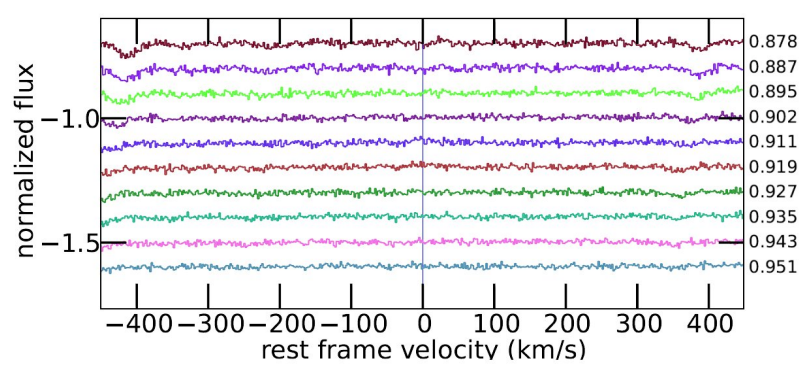

Fig. 8. Evolution of the He II $\lambda 4685.68$ line profile of RR Lyr. The intensity of the expected $\mathrm{He}$ II emission at $\varphi=0.911$ and 0.919 is $0.4 \%$ and $0.2 \%$ above the continuum, respectively.

in non-Blazhko stars. Preston notes that He II emission appears during pulsation cycles in which the metallic line doubling occurred. As far as our studies of RR Lyr are concerned, we have not observed an effective metallic line doubling but rather a line broadening (on iron lines, for example). This means that the shock velocity was not yet high enough to produce the complete line doubling. This is consistent with the presence of a very faint He II emission in our observations.

\section{Physical origin of helium emissions}

As discussed in the previous section, the helium emission occurs when the shock intensity is high enough to also induce a metallic line doubling or a line broadening. Thus, when the shock Mach number is more than a critical value, which depends on the upstream temperature of the atmosphere and the shock front velocity, helium appears in emission. Consequently, the observation of helium emission is the signature of a strong shock in the atmosphere. Because helium emission has never been observed in non-Blazhko stars, we can conclude that shock intensities in unmodulated RR Lyrae are always weaker than ones in Blazhko variables. Thus, a specific mechanism must exist in Blazhko stars, which amplifies the shock intensity.

The presence or the absence of helium emission lines can be considered as indicating the shock intensity. Equally, if the metallic lines do not reveal a doubling, but only present a linewidth broadening, this may also give us a clue to the strength of the shock. The minimum energy required for exciting a neutral helium atom is $19.73 \mathrm{eV}$. Because of the tail of the 
Maxwellian distribution, this means that if we observe helium emission lines, then the temperature in the de-excitation region of the shock wake is higher than $10000 \mathrm{~K}$, typically $20000 \mathrm{~K}$ when the He I and H I line strength are similar. Thus, we can define a first threshold Mach number $M_{\mathrm{He}}$.

Moreover, the observation of He II at $\lambda 4685.68$, which is supposed to form in the shock wake, requires that the minimal temperature would be higher than $30000 \mathrm{~K}$, typically around $40000 \mathrm{~K}$ to have a line strength greater than for He I lines. Therefore, the observation of He II emission indicates that the shock Mach number reaches a second critical value, which refers to the ionization of a neutral helium atom (minimum energy required $24.59 \mathrm{eV}$ ). It can be considered as a second threshold Mach number $M_{\mathrm{He} \text { II }}$. Observations show that only very few RR Lyrae stars are affected by such strong shock waves.

Finally, we can define three main shock regimes: (a) the supersonic regime in which only hydrogen emissions are visible, (b) the weak hypersonic regime where the He I emission lines are produced, and (c) the strong hypersonic regime with the additional observation of He II emission lines.

\section{Occurrence of helium emissions induced by the Blazhko effect?}

Gillet (2013) proposes a shock amplification mechanism to explain the Blazhko effect. All RRab pulse in the fundamental mode that produces the so-called "main shock". According to the analysis of Fokin \& Gillet (1997), the main shock is composed of shocks s1 and s2 caused by the $\kappa$-mechanism occurring in hydrogen and helium subphotospheric ionization layers, respectively. Above the photosphere, s2 merges with s1 into a single shock: the main shock. In the explanation of the Blazhko effect proposed by Gillet (2013), it is assumed that the pulsation of Blazhko stars are also affected by the transient first overtone. A shock s3' is created and may be the consequence of the perturbation of the fundamental mode by the first overtone. This shock of moderate intensity crosses the photosphere at the pulsation phase $0.5-0.6$, i.e., after the onset of the free-falling motion of the highest atmospheric layers. The role of s3' is to gradually slow the free-falling motion of the atmosphere down, pulsation cycle after pulsation cycle. Thus the effect of s3' is to decrease the atmospheric compression at each pulsation cycle somewhat, and therefore the intensity of the $\kappa-\gamma$-mechanism. When radiative losses of the main shock become strong, the average effective temperature of the star slightly decreases and the star begins a small redward journey on the Hertzsprung-Russell diagram. Consequently, the effect of the first overtone on the fundamental mode also decreases, like the intensity of s3', and as a result, the intensity of the main shock increases more and more. This dynamical amplification process takes place until the synchronized motion of photospheric layers breaks down. Thus, depending on star parameters, the shock intensity can be strong enough to excite He I, and for the strongest shocks, ionize He I and excite He II. Because the atmosphere of non-Blazhko stars is not altered by the first overtone, this amplification process cannot take place, and the intensity of the main shock is comparatively moderate and relatively stable. In any case, up until now, helium emission lines in non-Blazhko stars have never been reported. No amplification process of the shock intensity in these stars may be expected.
Finally, helium emissions are produced within the hot wake of the main shock when its intensity is sufficiently high. The maximum intensity of helium emissions occur exactly at the same pulsation phase as that of hydrogen emission lines ( $\varphi=0.911$ during our observation done on the July 4, 2011). This is quite consistent with the expected shock origin. It is not yet clear whether the desynchronization of photospheric layers can occur when the intensity of the main shock only allows the emission of He I, but not those of He II. Further observations are required to clarify this point.

\section{Conclusion}

In this paper, the observation of helium emission lines in RR Lyr itself, the prototype of the RR Lyrae stars, is reported for the first time. Emission lines of neutral helium He I at $\lambda 6678.16$ (singlet) and $\lambda 5875.66$ (triplet) are clearly observed. Their maximum intensity with respect to the continuum are $7 \%$ and $2 \%$, respectively $(\varphi=0.911)$. A very weak He II emission at $\lambda 4685.68$ is expected to be detected at the same phase. From recent spectroscopic observations conducted by Preston (2009, 2011), it appears that helium emissions would only be observed in Blazhko stars with the highest amplitude. Until now, helium emission lines have never been observed in non-Blazhko stars. According to the explanation of the Blazhko effect proposed by Gillet (2013), the intensity of the main shock in non-Blazhko stars would never be strong enough to produce helium emission. The presence of neutral helium in emission is therefore an indication that the main shock wave is strong (weak hypersonic regime). If ionized helium emission $\lambda 4685.68$ is also observed, it means that the intensity of the shock is considerable (strong hypersonic regime).

Acknowledgements. Spectropolarimetric data have been reduced with the LibreESpRIT software written by J.-F. Donati (IRAP-Toulouse, France). We thank the French PNPS/INSU-CNRS for financial support. We gratefully acknowledge Joanne O'Donnell and Joli Adams for their very careful reading of the final version of this paper.

\section{References}

Abt, H. A. 1959, ApJ, 130, 824

Blazhko, S. 1907, Astron. Nachr., 175, 325

Buchler, J. R., \& Kollath, Z. 2011, ApJ, 731, 24

Chadid, M., \& Gillet, D. 1996a, A\&A, 308, 481

Chadid, M., \& Gillet, D. 1996b, A\&A, 315, 475

Donati, J.-F., Semel, M., Carter, B. D., Reed, D. E., \& Collier, Cameron, A. 1997, MNRAS, 291, 658

Fokin, A. B., \& Gillet, D. 1997, A\&A, 325, 1013

Gillet, D. 2013, A\&A, accepted

Gillet, D., \& Crowe, R. A. 1988, A\&A, 199, 242

Kolenberg, K., Bryson, S., \& Szabo, R. 2011, MNRAS, 411, 878

Kovtyukh, V. V., Wallerstein, G., Andrievsky, S. M., et al. 2011, A\&A, 526, A116

Le Borgne, J.-F., Klotz, A., \& Boer, M. 2007, IBVS, 5790, 1

Lèbre, A., \& Gillet, D. 1991, A\&A, 251, 549

Preston, G. W. 2009, A\&A, 507, 1621

Preston, G. W. 2011, AJ, 141, 6

Preston, G.W., Smak, J., \& Paczyński, B. 1965, ApJS, 12, 99

Schwarzschild, M. 1952, Transaction of the IAU VIII, ed. P. Th. Oosterhoff (Cambridge University Press), 811

Stothers, R. B. 2006, ApJ, 652, 643

Struve, O. 1947, PASP, 59, 192

Struve, O. 1948, AJ, 53, 108

Struve, O., \& Blaauw, A. 1948, ApJ, 108, 60

Wallerstein, G. 1959, ApJ, 130, 560

Whitney, C. 1956, AnAp, 19, 34 